\title{
Tecnura
}

INVESTIGACIÓN

\section{Efecto de la utilización de harina de Lens culinaris como extensor en las características físicas y aceptabilidad de una salchicha}

\section{Effect of the use of Lens culinaris flour as an extender in the physical characteristics and acceptability of a sausage}

\author{
José David Torres González¹, Kevin José González Morelo², Diofanor Acevedo Correa³, \\ José del Carmen Jaimes Morales ${ }^{4}$
}

Fecha de recepción: 10 de febrero de 2016

Fecha de aceptación: 15 de mayo de 2016

Cómo citar: Torres González, J. D., González Morelo, K. J., Acevedo Correa, D., \& Jaimes Morales, J. D. C. (2016). Efecto de la utilización de harina de Lens culinaris como extensor en las características físicas y aceptabilidad de una salchicha. Revista Tecnura, 20(48), 15-28. doi: 10.14483/udistrital.jour.tecnura.2016.3.a01

\section{Resumen}

Objetivo: En esta investigación se utilizó harina de Lens culinaris variedad verdina como extensor carnico y se analizó su efecto en las características físicas y aceptabilidad de una salchicha.

Metodología: Se obtuvo la harina, analizaron las características bromatológicas, el índice de absorción de lípidos (IAL) y la capacidad de retención de agua (CRA). Se elaboraron las salchichas y añadiendole un $5 \%$ de la harina, posteriormente al producto terminado se le evaluaron los parámetros bromatológicos, microbiológicos, texturales y sensoriales por triplicado.

Analisis estadistico: Se utilizó un diseño experimental completamente al azar, realizando tres replicas de la formulacion con harina de L. culinaris y se efectaron análisis de varianza para hallar las diferencias estadísticas.

Resultados: El contenido de proteína de la harina fue $35,89 \%$ mientras que el de fibra $11,82 \%$. La capacidad de retención de agua y el índice de absorción de lípidos para la harina fueron de 3,87 mL agua/g muestra y 2,01 mL de aceite/g muestra. El producto final presentó recuentos microbianos que estuvieron dentro de lo establecido por las Normas Técnicas Colombianas. La harina influyó positivamente en la aceptabilidad de las salchichas y fue favorable en todos los parámetros de textura, lo cual fue similar a lo obtenido en salchichas comerciales. Conclusiones: La harina de L. culinaris representa una alternativa como materia prima no cárnica en la elaboración de productos con interesantes valores proteicos. Estos resultados pueden incentivar la utilización de la harina de lenteja de la variedad verdina en productos alimenticios de consumo masivo. Financiamiento: Grupo de Investigación Nutrición Salud y Calidad Alimentaria, Universidad de Cartagena.

Palabras clave: Alimento, funcionalidad, nutrición, proteína

\footnotetext{
Ingeniero de Alimentos, magíster en Ciencias Agroalimentarias, estudiante de doctorado en Ingeniería Química y Bioprocesos. Santiago de Chile, Chile. Contacto: jtorresg3@unicartagena.edu.co

2 Ingeniero de Alimentos, auxiliar de investigación grupo de Investigación Nutrición, Salud y Calidad Alimentaria. Cartagena, Colombia. Contacto: kevinjgonza@hotmail.com

3 Ingeniero de Alimentos, especialista en Ciencia y Tecnología de Alimentos, doctor en Ingeniería. Docente de la Universidad de Cartagena. Cartagena, Colombia. Contacto: diofanor3000@gmail.com

$4 \quad$ Ingeniero de Alimentos, magíster en Ciencia y Tecnología de Alimentos, candidato a doctor en Ciencias. Docente de la Universidad de Cartagena. Cartagena, Colombia. Contacto: jjaimesmor@yahoo.es
} 


\section{Abstract}

Objective: The objective of this research was to use Lens culinaris flour, verdina variety, as a meat extender, and to analyze its effect on the physical characteristics and acceptability of a sausage.

Methodology: The bromatological characteristics, oil absorption index (OAI) and water holding capacity (WHC) were analyzed on the obtained flour. The sausage was prepared, it was added $5 \%$ of flour to it; then, the bromatological, microbiological, textural, and sensory parameters per triplicate of the finished product were evaluated.

Statistical Analysis: An experimental design was used completely at random, making three replicates of the formulation, and analysis of variances was performed to find statistical differences.

Results: The protein content of the flour was 35.89 $\%$ while the fiber was $11.82 \%$. The water holding capacity and the lipid index absorption were 3.87 $\mathrm{mL}$ water/g sample, and $2.01 \mathrm{ml}$ of oil/g sample. The final product showed that microbial counts were within the provisions of the Colombian Technical Standards. Flour positively influenced the acceptability of sausages and was favorable in all texture parameters, which were similar to those obtained in commercial sausages.

Conclusions: L. culinaris flour represents a non-meat alternative raw material in the manufacture of products with a good protein source. These results may encourage the use of Lentil flour with verdina variety of food products consumers.

Financing: the Nutrition, Health and Food Quality Research Group, University of Cartagena.

Keywords: Food, functionality, nutrition, protein.

\section{INTRODUCCIÓN}

En la actualidad ha surgido un gran interés por el uso de nuevas fuentes de proteínas, debido al aumento exponencial de la población, lo que a su vez genera un déficit creciente a escala mundial de productos proteicos (Liu et al., 2008). Con el fin de satisfacer esta demanda, se han realizado numerosas investigaciones tendientes a encontrar nuevas tecnologías que faciliten un mayor aprovechamiento de las fuentes de proteínas existentes (Boye et al., 2010). Colombia se encuentra entre los países de mayor biodiversidad de alimentos y materias primas vegetales; sin embargo, la desnutrición sigue siendo uno de los grandes problemas que afecta la salud de la población, en especial la falta de proteínas en la dieta de los niños, que se manifiesta con problemas de crecimiento, enfermedades y pérdidas en el desarrollo cerebral (Restrepo et al., 2010). Según la última encuesta de situación nutricional en Colombia, la deficiente ingesta de proteínas alcanza el 36,6 \% lo cual significa que lo recomendado $(0,91 \mathrm{~g} / \mathrm{kg}$ de peso) no está satisfecha en un gran sector de la población (Restrepo et al., 2010; Sánchez-Zapata et al., 2011; Montero et al., 2015). Estas deficiencias son más marcadas en los grupos poblacionales de escasos recursos, debido al elevado costo y la poca disponibilidad de los productos proteicos en cualquier etapa del año (Benjumea, Estrada y Álvarez, 2006). Las leguminosas tienen un elevado contenido proteico (20-45\%) y pueden ser considerados como un complemento natural a los cereales, ya que contienen cantidades adecuadas de lisina $(\mathrm{Ol}-$ medilla, Ferré-Rovir, Asencio-Vegas y Matín-Pedrosa, 2010). Algunas leguminosas, como la soya, fríjol común, Zaragoza, garbanzo, cacahuete, habas y arvejas, pueden producir flatulencias, y otras como lupinus y el fríjol mucuna, contienen principios tóxicos que normalmente son eliminados al someterlas a cocción por ebullición (Boye et al., 2010; Zhang et al., 2010).

Las lentejas (Lens culinaris) son legumbres que crecen en vainas, la cual contiene semillas en su interior; son bastante pequeñas, planas y son similares a los guisantes (Joshi, Adhikari, Panozzo 
y Aldred, 2010). Existen cientos de variedades de lentejas que difieren principalmente por sus propiedades fisicoquímicas y en el color (Gharibzahedi, Emam-Djomeh, Razavi y Jafari, 2014); se pueden encontrar en amarillo, naranja, rojo, verde, marrón y negro, hoy en día se consumen en todo el mundo. Algunos estudios revelan que las lentejas reducen el colesterol (Oroian, 2015) debido a la fibra soluble que se une a este, por lo cual se reducen los niveles en la sangre (Gharibzahedi et al., 2014). Las lentejas son ricas en fósforo, también tienen una alta cantidad de vitaminas B1, B2, B3, B5, B6, B9, A, C, K, E, además de grandes cantidades de minerales como potasio, hierro, calcio, yodo, zinc, sodio, y de componentes como fibra, proteínas, carbohidratos (Oroian, 2015).

Con el propósito de disminuir los costos de producción en la formulación de los productos cárnicos, se han introducido algunas sustancias denominadas extensores, con el propósito de sustituir una parte de la carne que se emplea y así ofrecer el aporte proteico y funcional adecuado (Utrilla, García-Ruiz y Soriano, 2014). Sin embargo, no es recomendable la utilización excesiva de los extensores, porque esto puede influir negativamente en la calidad y aceptabilidad del producto final (Rodríguez, Amorrortu y Álvarez, 2011; Guavita, 2012). Por ende es necesario establecer proporciones adecuadas para cada derivado cárnico a elaborar (Utrilla, García-Ruiz y Soriano, 2014). Las harinas, concentrados y aislados de cereales y legumbres son ejemplos de extensores cárnicos más usados en la industria alimentaria (Achouri et al., 2010). Cabe resaltar que el uso de extensores, además de proporcionar proteínas de alto valor biológico, sustituyen parcialmente la proteína cárnica y otros nutrimentos asociadas a esta (Boye et al., 2010; Jaimes et al., 2015). Los extensores cárnicos tienen propiedades funcionales como la retención de agua, la emulsificación de las grasas, absorción de lípidos y la gelificación (Olmedilla-Alonso, Jiménez-Colmenero y Sánchez-Muniz, 2013). Así la presencia de uno u otro extensor no solo ayuda en la parte económica a la fórmula, sino que además concede rendimiento al proceso y al producto como tal (Arief et al., 2014; Marchetti, Andrés y Califano, 2014).

Teniendo en cuenta lo anterior, el objetivo del presente trabajo fue utilizar harina de Lens culinaris variedad verdina, como extensor cárnico, y analizar su efecto en las características bromatológicas, microbiológicas, texturales y aceptabilidad de un producto cárnico tipo salchicha.

\section{MATERIALES Y MÉTODOS}

\section{Obtención de la muestra}

Se tomó una muestra de $4000 \mathrm{~g}$ de $L$. culinaris adquiridas de un supermercado local de la ciudad de Cartagena de Indias (Bolívar, Colombia). Esta cantidad fue suficiente para la obtención de la harina, realizar las pruebas y elaborar las salchichas. Las semillas se seleccionaron teniendo en cuenta que estuvieran sanas (enteras y sin picaduras de gorgojo); se secaron utilizando una incubadora a 40 ${ }^{\circ} \mathrm{C}$ (incubadora Thermolyne). Luego molidas en un molino tradicional, el producto se pasó por un tamiz de malla No. 40 hasta obtener una harina fina (Jaimes, Restrepo y Acevedo, 2014).

\section{Evaluación de la composición de la harina}

\section{Análisis bromatológicos}

A la harina se le realizaron determinaciones de humedad (No. 952,08), grasa cruda (No. 948,15), cenizas (No. 942,05), proteína cruda según el método de Kjeldahl con factor 6,25 (No. 995,04), fibra (No. 962,09) siguiendo la metodología propuesta por la AOAC (2005). Una vez obtenidos todos estos valores, la diferencia se tomó como el porcentaje de carbohidratos totales a través de la ecuación (1). Las calorías (kcal/100 g) se determinaron de manera similar a lo realizado por Alvis, Vélez y Rada-Mendoza (2008) mediante la relación de los porcentajes de proteínas, grasa y carbohidratos presentada en la ecuación (2). La harina de Lens culinaris fue comparada con resultados de 
otras investigaciones donde utilizaron diferentes harinas de leguminosas y cereales.

Carbohidratos $=100-(\%$ de humedad $)$

$+(\%$ de proteína $)+(\%$ de grasa $)+(\%$ de ceniza)

Calorías $(\mathrm{kcal} / 100 \mathrm{~g})=(4 \times \%$ de proteína $)+(9 \times \%$ de grasa $)+(4 \times \%$ de carbohidratos)

\section{Propiedades funcionales a la harina de $\mathbf{L}$. culinaris}

Índice de absorción de lípidos (IAL)

Representa la cantidad de aceite por $100 \mathrm{~g}$ de harina. Se determinó agregando un exceso de aceite $(3 \mathrm{ml})$ a la muestra $(0,5 \mathrm{~g})$ en tubos graduados de centrífuga; posteriormente se agitó por 1 minuto; luego se colocó a $24{ }^{\circ} \mathrm{C}$ durante 30 minutos y se centrifugó a 3200 revoluciones por minuto y se midió el volumen de aceite excedente. La densidad del aceite fue de 0,86 g/ml. Se calculó el índice de absorción de lípidos (\% de IAL) mediante la ecuación (3) (Bermúdez, 1994; Granito, Pérez y Suhey, 2009).

$\%$ de $I A L=(\mathrm{ml}$ aceite absorbido/gra-

mos de muestra) $\times 100$

\section{Capacidad de retención de agua (CRA)}

Expresa la cantidad de agua retenida por $100 \mathrm{~g}$ de harina. Se cuantificó, colocando 0,5 g de muestra en un tubo de centrífuga, agregando un exceso de agua $(3 \mathrm{ml})$. Se agitó por 1 minuto. Los tubos se centrifugaron a 3200 revoluciones por minuto, después de haberse mantenido a $24{ }^{\circ} \mathrm{C}$ por $30 \mathrm{mi}$ nutos; para medir el volumen de agua no retenida, se empleó la ecuación (4) (Bermúdez, 1994; Granito, Pérez y Suhey, 2009).

$\%$ de $C R A=(\mathrm{ml}$ de agua retenida/gramos de muestra) $\times 100$

\section{Elaboración de la salchicha}

Se utilizó carne de res y de cerdo que se compró y trasladó a las instalaciones del Grupo de Investigación Nutrición, Salud y Calidad Alimentaria, de la Facultad de Ingeniería en la Universidad de Cartagena, donde se pesó y, posteriormente, se separó de la grasa y de otros componentes como el hueso y la piel. La carne fue picada en una picadora de discos de $5 \mathrm{~mm}$ de espesor, y se colocó en una maquina Cutter, con el fin de disminuir el tamaño de partícula. En esta etapa de procesamiento se agregó la mitad del hielo a procesar, los condimentos y la sal, teniendo en cuenta los porcentajes establecimientos por las Normas Técnicas Colombianas (Hleap y Velasco, 2012).

Posteriormente la grasa fue agregada, al igual que la otra mitad del hielo y la harina L. culinaris. La pasta se retiró de la maquina Cutter, una vez que esta adquirió homogeneidad. El mezclado se realizó por 15 minutos y la temperatura de la masa final del proceso no fue mayor a $15{ }^{\circ} \mathrm{C}$ para evitar que las proteínas se desnaturalizaran y, por ende, perdieran su capacidad de retener agua.

Una vez obtenida la emulsión, se efectuó el embutido, el cual se llevó a cabo en una embutidora hidráulica, utilizando tripas naturales. El amarrado se hizo manualmente y en el cual se garantizó un peso de 60 gramos, correspondiente al peso comercial; las salchichas fueron sometidas a escaldado, donde se efectuó la coagulación de las proteínas. Esta etapa tecnológica se realizó en un tanque de cocción rectangular, con agua corriente a temperatura de $95 \pm 1{ }^{\circ} \mathrm{C}$, hasta que la temperatura medida con una termocupla en una salchicha tomada aleatoriamente alcanzó los $72 \pm 1{ }^{\circ} \mathrm{C}$. Para disminuir la temperatura de las salchichas y lograr una coagulación correcta; estas fueron sometidas a un choque térmico, para lo cual se utilizó una mezcla de agua y hielo a temperatura de $10 \pm 2{ }^{\circ} \mathrm{C}$, durante aproximadamente cinco minutos. Las salchichas se almacenaron y refrigeraron a $4{ }^{\circ} \mathrm{C}$. En la tabla 1 se detalla la formulación utilizada para elaborar las salchichas con harina de L. culinaris. 
Tabla 1. Formulación empleada para elaborar salchichas con harina de L. culinaris

\begin{tabular}{lcc}
\hline \multicolumn{1}{c}{ Ingredientes } & Formulación $\mathbf{( g )}$ & $\mathbf{( \% )}$ \\
\hline Carne de res & 480 & 48,0 \\
\hline Carne de cerdo & 120 & 12,0 \\
\hline Grasa & 200 & 20,0 \\
\hline Hielo & 135 & 13,5 \\
\hline Harina de L. culinaris & 50 & 5,0 \\
\hline Consomé de pollo & 2 & 0,16 \\
\hline Cebolla en polvo & 1 & 0,10 \\
\hline Pimienta blanca molida & 3 & 0,30 \\
\hline Glutamato monosódico & 1 & 0,10 \\
\hline Ajo en polvo & 1 & 0,10 \\
\hline Sal & 2 & 0,24 \\
\hline Humo líquido & 5 & 0,50 \\
\hline Total & 1000 & 100,0 \\
\hline
\end{tabular}

Fuente: elaboración propia.

\section{Evaluación de las características y aceptabilidad de las salchichas}

\section{Análisis bromatológicos}

Estos se realizaron de manera similar a los efectuados a la harina, en cuanto al contenido de humedad, cenizas, proteínas, grasa, fibra y carbohidratos (AOAC, 2005). Las muestras de salchichas se mantuvieron a $15^{\circ} \mathrm{C}$, todos los ensayos se realizaron por triplicado empleando diez gramos en cada caso.

\section{Análisis sensorial}

Se llevó a cabo por 20 panelistas semientrenados, en una habitación apropiada para tal fin. Los miembros indicaron cuánto les gustaba o disgustaba el producto, para lo cual se utilizó una escala hedónica de 5 puntos (excelente, buena, neutral, regular, mala) en cuanto a las características de olor, color, sabor, dureza y aceptabilidad general, similar a lo realizados por Hleap, Gutiérrez, Rivera (2010), y Granados, Guzmán y Acevedo (2013). Los datos de cada panelista se recogieron en una planilla para su análisis posterior; los análisis fueron comparados con los realizados a una salchicha comercial, elaborada con harina de soya.

\section{Análisis microbiológicos}

Se realizaron pruebas de conteo total de aerobios mesófilos, coliformes totales y fecales, Staphylococcus coagulasa positivos, Salmonella y Escherichia coli a las salchichas escaldadas, según la metodología indicada por la Norma Técnica Colombiana (Icontec, 2008). Las determinaciones se hicieron por triplicado empleando $15 \mathrm{~g}$ en cada caso y los datos se expresaron como $\log _{10}$ de UFC/g.

\section{Análisis de perfil de textura (TPA)}

De acuerdo con la metodología planteada por Montero, Acevedo, Arnedo y Miranda (2015) y Torres, Acevedo y Tirado (2015). Se cortaron las salchichas elaboradas con harina de L. culinaris, en rodajas de 1,5 cm de espesor y se dejaron en reposo por un lapso de una hora dentro de una bolsa de polietileno, con el fin de evitar la pérdida de humedad de las muestras. Se utilizó un texturómetro marca TA.TX2 $\mathrm{i}^{\circledR}$ Plus Stable Micro System, acoplado al programa Texture Expert Exceed, versión 2.64. Se realizó una doble compresión uniaxial a $75 \%$ de deformación (estrés normal) y a una velocidad de cabeza de $2 \mathrm{~mm} / \mathrm{s}$, con un tiempo de espera de 5 segundos entre las compresiones. Se determinaron los siguientes parámetros de textura: dureza $(\mathrm{N})$, adhesividad $(\mathrm{N})$, cohesividad (adimensional), elasticidad (adimensional) y masticabilidad (kg). Estos análisis al igual que los sensoriales y bromatológicos se compararon con los obtenidos de una salchicha comercial elaborada con harina de soya.

\section{Análisis estadístico}

En esta investigación se empleó un diseño experimental completamente al azar (DCA) debido a que todos los análisis respectivos fueron hechos en laboratorio bajo condiciones controladas de temperatura y humedad ambiente. La formulación de la salchicha con harina de $L$. culinaris fue realizadas tres veces, es decir, se tuvieron tres réplicas del experimento. Todos los análisis pertenecientes a cada réplica se realizaron por triplicado y se 
registró el promedio aritmético y su desviación estándar. El procesamiento de la información obtenida se llevó a cabo con el programa estadístico Statgraphics Centurion ${ }^{\circledR}$ versión 16.0 en Windows 10, en el cual se determinó la existencia o no de diferencias estadísticamente significativas de cada uno de los parámetros evaluados en los ensayos, a través de un análisis de varianza (ANOVA) completamente aleatorio y mediante comparaciones múltiples, empleando el test HSD de Tukey con un nivel de significancia del $5 \%(p \leq 0,05)$.

\section{RESULTADOS}

En la tabla 2 se muestra la composición bromatológica obtenida de la harina de L. culinaris. Esta fue comparada con valores reportados por otros autores en harinas leguminosas y cereales, utilizados comúnmente en la elaboración de embutidos cárnicos. El contenido de humedad de la harina $L$. culinaris fue de $6,38 \pm 0,61 \%$, sin encontrarse diferencias estadísticamente significativas ( $p>0,05)$ con respecto a la harina de soya $5,98 \pm 0,59 \%$, la cual es muy frecuente en la elaboración de productos cárnicos. Por su parte, las harinas de fríjol $(12,01 \pm 0,31 \%)$, trigo $(12,11 \pm 0,33 \%)$ y quinua $(11,74 \pm 0,01 \%)$ mostraron los mayores porcentajes de humedad, siendo diferente estadísticamente $(\mathrm{p}<0,05)$ respecto a la harina de $L$. culinaris.

El contenido de proteínas para la harina de soya fue de $34,81 \pm 0,39 \%$ mientras que para la harina

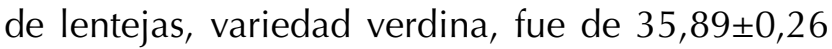
$\%$ y no hubo diferencias significativas entre estos valores, lo que lleva a considerar la importancia de la harina de lentejas para la elaboración de productos proteicos. Es pertinente resaltar la importancia de desarrollar investigaciones donde se indique el valor biológico y el tipo de proteínas presentes en dicha harina. En cuanto al contenido lipídico se observó mucha variabilidad de los valores reportados en los diferentes tipos de harinas, respecto a la de lenteja $(p<0,05)$, debido a que las de chachafruto, fríjol, trigo y quinua, aquí mencionadas, fueron desengrasadas durante su elaboración, según los respectivos investigadores. La harina de la leguminosa promisoria tarwi,

Tabla 2. Composición bromatológica de la harina de L. culinaris y comparación con algunas leguminosas y cereales utilizados en la elaboración de salchichas

\begin{tabular}{lccccccc}
\hline \multicolumn{1}{c}{$\begin{array}{c}\text { Componentes } \\
\text { harinas }(*)\end{array}$} & $\begin{array}{c}\text { Humedad } \\
(\%)\end{array}$ & $\begin{array}{c}\text { Proteínas } \\
(\%)\end{array}$ & $\begin{array}{c}\text { Lípidos } \\
(\%)\end{array}$ & Fibra (\%) & $\begin{array}{c}\text { Cenizas } \\
(\%)\end{array}$ & $\begin{array}{c}\text { Carbohidratos } \\
(\%)\end{array}$ & $\begin{array}{c}\text { Calorías } \\
(\mathbf{k c a l} / \mathbf{1 0 0 g})\end{array}$ \\
\hline Lentejas & $6,38 \pm 0,61 \mathrm{a}$ & $35,89 \pm 0,26 \mathrm{~d}$ & $1,25 \pm 0,07 \mathrm{~b}$ & $11,82 \pm 0,30 \mathrm{c}$ & $3,01 \pm 1,34 \mathrm{c}$ & $53,47 \pm 8,38 \mathrm{~b}$ & $374,53 \mathrm{a}$ \\
\hline $\begin{array}{l}\text { Soya (Delgado y } \\
\text { Albarracín, 2012) }\end{array}$ & $5,98 \pm 0,59 \mathrm{a}$ & $34,81 \pm 0,39 \mathrm{~d}$ & $9,54 \pm 0,07 \mathrm{~d}$ & $7,59 \pm 0,42 \mathrm{~b}$ & $4,68 \pm 0,23 \mathrm{~cd}$ & $44,99 \pm 7,67 \mathrm{~b}$ & $405,06 \mathrm{e}$ \\
\hline $\begin{array}{l}\text { Chachafruto (Del- } \\
\text { gado y Albarracín, } \\
\text { 2012) }\end{array}$ & $9,01 \pm 0,60 \mathrm{c}$ & $22,81 \pm 0,28 \mathrm{~b}$ & $0,70 \pm 0,01 \mathrm{a}$ & $\mathrm{NA}$ & $5,35 \pm 0,52 \mathrm{e}$ & $62,14 \pm 1,87 \mathrm{~d}$ & $346 \mathrm{~b}$ \\
\hline $\begin{array}{l}\text { Tarwi (Castañeda } \\
\text { et al., 2009). }\end{array}$ & $7,72 \pm 0,42 \mathrm{~b}$ & $44,31 \pm 0,37 \mathrm{e}$ & $16,50 \pm 0,54 \mathrm{e}$ & $7,11 \pm 0,35 \mathrm{~b}$ & $3,34 \pm 0,72 \mathrm{c}$ & $28,13 \pm 0,34 \mathrm{a}$ & $438 \mathrm{f}$ \\
\hline $\begin{array}{l}\text { Fríjol (Granito et al., } \\
\text { 2009). }\end{array}$ & $12.01 \pm 0,31 \mathrm{~d}$ & $26,24 \pm 0,36 \mathrm{c}$ & $1,66 \pm 0,51 \mathrm{~b}$ & $3,33 \pm 0,62 \mathrm{a}$ & $3,62 \pm 0,38 \mathrm{c}$ & $56,47 \pm 1,23 \mathrm{c}$ & $387 \mathrm{~d}$ \\
\hline $\begin{array}{l}\text { Trigo (Goesaert et al., } \\
\text { 2005). }\end{array}$ & $12,11 \pm 0,33 \mathrm{~d}$ & $12,21 \pm 0,34 \mathrm{a}$ & $1,12 \pm 0,08 \mathrm{~b}$ & $3,62 \pm 0,81 \mathrm{a}$ & $0,84 \pm 0,02 \mathrm{a}$ & $73,72 \pm 1,65 \mathrm{e}$ & $348 \mathrm{~b}$ \\
\hline $\begin{array}{l}\text { Quinua (Delgado y } \\
\text { Albarracín, 2012) }\end{array}$ & $11,74 \pm 0,01 \mathrm{~d}$ & $11,84 \pm 0,64 \mathrm{a}$ & $2,05 \pm 0,01 \mathrm{c}$ & $\mathrm{NA}$ & $1,74 \pm 0,05 \mathrm{~b}$ & $72,63 \pm 0,08 \mathrm{e}$ & $356,33 \mathrm{c}$ \\
\hline
\end{tabular}

*Valores expresados en $100 \mathrm{~g}$ de base seca-Superíndices diferentes en una misma columna indican diferencias estadísticamente significativas $(p<0,05)$.

Fuente: elaboración propia. 
mostró la mayor cantidad de lípidos $(16,50 \pm 0,54$ \%) (Castañeda et al., 2009), seguida por la harina de soya, diferencia que fue significativa respecto a lo hallado en la harina de lentejas ( $<<0,05)$, la cual estuvo dentro de las más bajas. Estos resultados pueden incentivar la utilización de la harina de lenteja de la variedad verdina en productos alimenticios de consumo masivo bajos en grasa; aun así es importante la identificación y la cuantificación de los tipos de ácidos grasos que se encuentran en esta harina.

El contenido más alto de fibra lo presentó la harina de $L$. culinaris $(11,82 \pm 0,53 \%)$, seguido por la harina de soya $(7,59 \pm 0,42 \%)$, la cual es utilizada en productos cárnicos de consumo tradicional, así como en galletas y demás productos de molinería que se comercializan. Así se resalta la importancia del contenido elevado en fibra, lo que podría llevar a que se utilice la harina L. culinaris, variedad verdina, en este tipo de productos que brinden al consumidor los beneficios que aporta la fibra en la dieta.
En la harina de lentejas también se encontró un buen contenido de cenizas $(3,01 \pm 1,34 \%)$ comparable con la harina de soya $(4,68 \pm 0,23 \%)(p>$ $0,05)$, por lo cual sería necesario un estudio más detallado en cuanto al análisis de estos elementos, en esta variedad de lentejas verdes. Por otra parte, se observó un valor superior al de la soya en cuanto el contenido de carbohidratos, sin embargo, por ser harina proteica posee un contenido medio de carbohidratos $(53,47 \pm 8,38 \%$ y $44,99 \pm 7,67 \%$ ), en comparación con la harina de trigo $(73,72 \pm 1,65$ $\%)$, la quinua $(72,63 \pm 0,08 \%)$ y el chachafruto $(62,14 \pm 1,87 \%$ ) (Goesaert et al., 2005; Delgado y Albarracín, 2012).

En la figura 1 se encuentran los datos obtenidos del IAL = Índice de Absorción de Lípidos y capacidad de retención de agua (CRA), los cuales, a su vez, fueron comparados con otras investigaciones.

La capacidad de retención de agua de las harinas se relaciona en gran medida con el tamaño de las partículas, con la cantidad de grupos hidroxilos libres capaces de interactuar con el agua externa y con

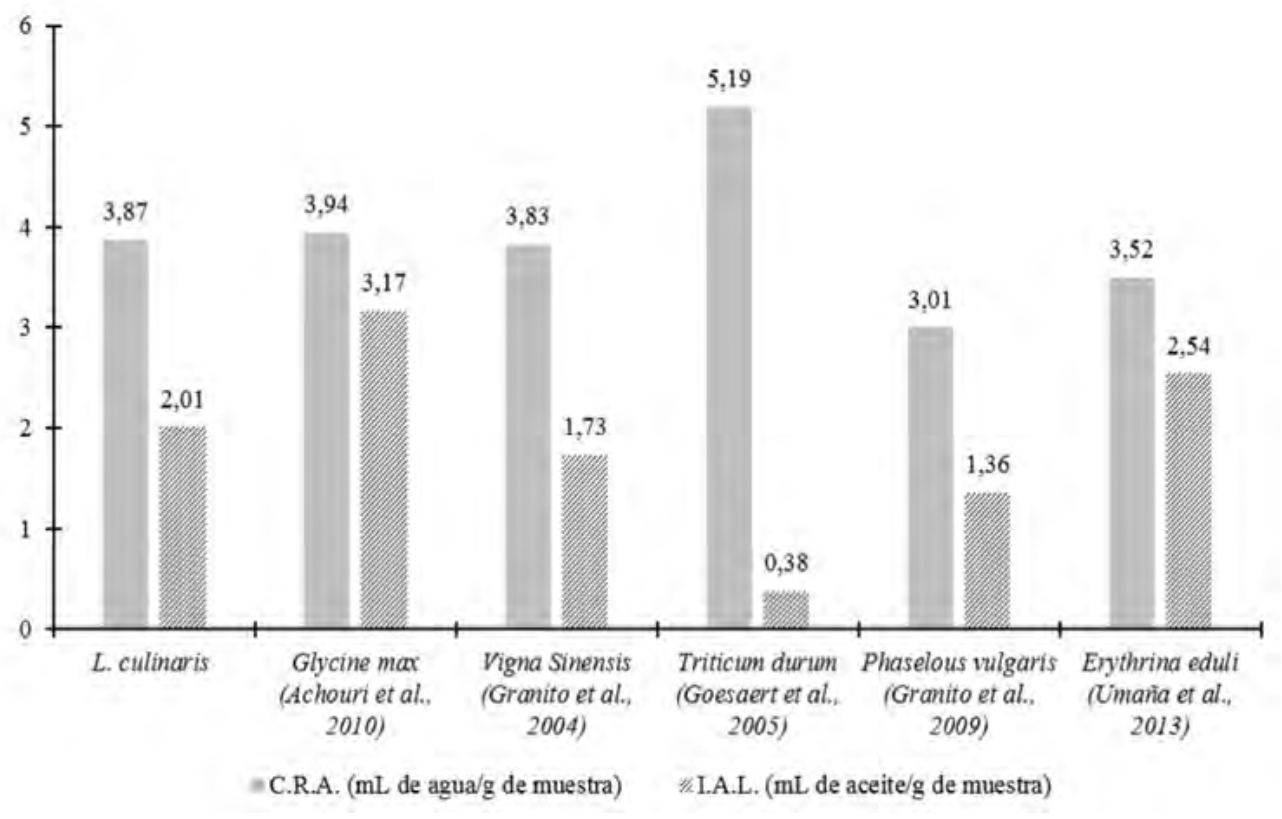

Figura 1. Propiedades funcionales de la harina de L. culinaris y otras de uso común en la elaboración de productos cárnicos tipo salchicha

Fuente: elaboración propia. 
la cantidad de fibra soluble de las mismas (Achouri et al., 2010; Umaña, Lopera y Gallardo, 2013).

El valor promedio de CRA reportado para la harina de trigo fue significativamente mayor al de la harina L. culinaris $(\mathrm{p}<0,05)$; esta diferencia puede ser atribuida a un mayor contenido de carbohidratos, ya que los azúcares libres tienden a hidratarse con mayor facilidad y a un menor tamaño de las partículas en estas harinas, pues teóricamente cuanto menor sea este, la facilidad de hidratación aumenta, debido a la superficie de contacto que se tiene (Goesaert et al., 2005; Granito et al., 2009). Una de las características que posee la CRA como propiedad funcional en productos cárnicos radica en su relación con las proteínas, macromoléculas que tienen la capacidad de interactuar con el agua formando puentes de hidrógeno por los grupos polares que estos compuestos tienen, proporcionando la capacidad de hidratación, hinchamiento, solubilidad, viscosidad y gelación (De la Torre-Gutiérrez, Chel-Guerrero, Betancur-Ancona, 2008).

En su estudio, Liu et al. (2008) indicaron que la CRA de una muestra de harina no es necesariamente una función de su contenido proteico, y que la presencia de componentes no proteicos puede también influir sobre esta. Por su parte, Oshodi y Ekperigin (1989) demostraron que el tratamiento térmico húmedo desnaturaliza las proteínas; debido a este efecto, incrementa la accesibilidad a dicha proteína y, en consecuencia, a los aminoácidos polares, los cuales tienen una gran afinidad por el agua, produciéndose un incremento en la CRA. Valores de absorción de agua mayores a 300 \% (3,0g de agua/g de muestra) pueden favorecer la textura de productos de panadería (Achouri et al., 2010) y además esta propiedad es importante en una variedad de alimentos cárnicos en los cuales se requiere retener agua durante su elaboración (Ambrosiadis, Soultos, Abrahim y Bloukas, 2004). Por tanto, la harina de L. culinaris, cuyo valor de CRA superó el mencionado, puede representar una alternativa importante para enriquecer mezclas de harinas en la elaboración galletas, tortas, panes, así como en la preparación de productos cárnicos.
El IAL, que se relaciona con el número de cadenas laterales no polares de las proteínas que se enlazan con cadenas hidrocarbonadas de grasa (Granito, Pérez y Suhey, 2009), es una propiedad importante en la formulación de embutidos cárnicos, en productos de panadería y sopas. Los lípidos pueden interactuar de diferentes maneras con las proteínas, básicamente a través de puentes hidrófobos (Achouri et al., 2010; Boye et al., 2010).

En productos cárnicos emulsionados, las proteínas de soya promueven la absorción y retención de grasa, por tanto se disminuyen las pérdidas durante la cocción y se mantiene la estabilidad dimensional. El IAL de la harina de $L$. culinaris fue de 2,01 $\mathrm{ml}$ de aceite/g de muestra; menor a lo reportado para la harina de Glycine $\max (3,17 \mathrm{ml}$ de aceite/g de muestra), pero mayor a la harina de Vigna Sinensis $(1,73 \mathrm{ml}$ de aceite/g de muestra), Triticum durum $(0,38 \mathrm{ml}$ de aceite/g de muestra) y Phaselous vulgaris (1,36 $\mathrm{ml}$ de aceite/g de muestra) y muy similar a la harina de Erthrina edulis $(2,54$ $\mathrm{ml}$ de aceite/g de muestra), lo cual es un indicador de que puede ser empleada en productos cárnicos, porque promueve la absorción y retención de grasa, lo que en consecuencia, podría ayudar a disminuir las pérdidas durante el escaldado y a mantener la estabilidad de la emulsión (Marchetti, Andrés y Califano, 2014). La habilidad para absorber y retener dichos compuestos es un indicativo de la capacidad que tienen las proteínas para enlazar agua, que influye drásticamente en la textura y las propiedades sensoriales de los productos a consumir (Goesaert et al., 2005). La absorción de aceite es atribuida a la retención física de la grasa por atracción capilar y al enlace que se forma con la cadena apolar proteica, de allí que la hidrofobicidad de las proteínas ejerza un papel importante sobre la absorción de la grasa (Jaimes, Restrepo y Acevedo, 2014). El conocimiento de la absorción de aceite es importante en la ingeniería de alimentos, ya que determina características del producto, como el sabor, la palatabilidad y la vida útil (Oshodi y Ekperigin, 1989). 
En la tabla 3 se esquematizan los resultados de los análisis bromatológicos obtenidos de la salchicha con harina de L. culinaris y la salchicha comercial elaborada con harina de soya.

Tabla 3. Composición bromatológica de la salchicha con harina de L. culinaris, respecto a una comercial

\begin{tabular}{lcc}
\hline $\begin{array}{c}\text { Componente } \\
(\mathbf{g} / \mathbf{1 0 0 g})^{*}\end{array}$ & $\begin{array}{c}\text { Salchicha con } \\
\text { L. culinaris }\end{array}$ & $\begin{array}{c}\text { Salchicha } \\
\text { comercial }\end{array}$ \\
\hline Humedad & $57,81 \pm 0,82 \mathrm{a}$ & $59,43 \pm 0,02 \mathrm{a}$ \\
\hline Ceniza & $3,71 \pm 0,09 \mathrm{a}$ & $3,65 \pm 0,17 \mathrm{a}$ \\
\hline Proteína & $18,33 \pm 0,66 \mathrm{a}$ & $17,98 \pm 0,09 \mathrm{a}$ \\
\hline Grasa & $16,13 \pm 0,44 \mathrm{a}$ & $16,36 \pm 0,09 \mathrm{a}$ \\
\hline Carbohidratos & $2,82 \pm 0,09 \mathrm{a}$ & $2.80 \pm 0,03 \mathrm{a}$ \\
\hline Fibra & $0,97 \pm 0,040$ & $\mathrm{ND}$ \\
\hline
\end{tabular}

*Letras diferentes en una misma fila indican diferencias significativas $(p<0,05)$; $N D=$ no detectada.

Fuente: elaboración propia.

En relación al contenido de proteínas para las salchichas en estudio y la salchicha patrón, no se observaron diferencias estadísticas $(18,33 \pm 0,66$ y $17,98 \pm 0,09)$, debido, posiblemente, a que las harinas de ambas leguminosas poseían un contenido de proteína similar.
Con respecto a la grasa de las salchichas evaluadas, el contenido se encontró dentro de los rangos reportados por otros autores como Dzudie, Scher y Hardy (2002), destacando en la salchicha con $L$. culinaris de $16,13 \pm 0,44$ y la salchicha comercial de 16,36 \pm 0,09g/100g de muestra. En relación al contenido de carbohidratos, no se observaron diferencias significativas con la salchicha patrón $(2,82 \pm 0,09$ y $2,80 \pm 0,03 \%)$. En cuanto al contenido de fibra dietética total, en la salchicha comercial no se registró, y en las salchichas con harina de

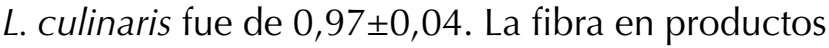
cárnicos es deseable porque el sabor es neutro y retiene agua, lo cual conserva la frescura del producto. Astaíza, Ruíz y Elizalde (2010) estudiaron la harina de quinua en pastas alimenticias, de lo cual lograron resultados satisfactorios en cuanto a calidad, composición nutricional y disminución de costos. Las proteínas se han utilizado en la elaboración de productos cárnicos con el fin de aumentar el rendimiento, las propiedades ligantes de agua, grasa y el valor nutritivo, así como cambiar las propiedades funcionales (Zhang et al., 2010).

En la figura 2, se muestran los resultados de los análisis sensoriales de las salchichas elaboradas con harina de L. culinaris, en cuanto a olor, sabor,

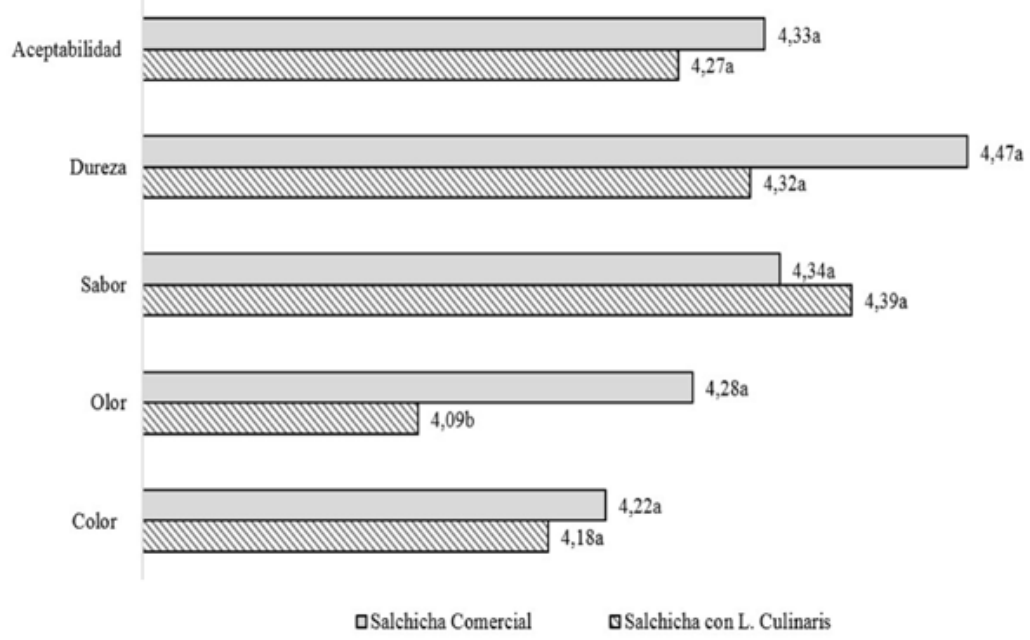

Figura 2. Aceptabilidad de las salchichas elaboradas con harina de L. culinaris, respecto a una salchicha comercial Fuente: elaboración propia. 
color, textura y aceptabilidad, y se compararon con una salchicha comercial. Cada característica representa los valores promedios de las calificaciones hechas por los panelistas. Se observa que la formulación con harina $L$. culinaris presentó en general buena aceptación general, con lo que se sobrepasó el umbral mínimo de aceptación de 3,0. La percepción del color y la dureza en la salchicha comercial fue ligeramente mayor que la salchicha con L. culinaris, sin embargo esta diferencia no fue estadísticamente significativa ( $p>0,05)$.

Al compararse el olor entre las muestras analizadas, se observó que el promedio para ambos productos sobrepasó la calificación de 4,0, siendo este puntaje ligeramente más alto en las salchichas comerciales, respecto a las elaboradas con harina de $L$. culinaris $(\mathrm{p}<0,05)$. Los panelistas indicaron una mejor percepción del sabor en las salchichas elaboradas con harina de lentejas, lo cual indica que la adición de este producto fue favorable en la calidad organoléptica de los embutidos. En la tabla 4 se detallan los datos obtenidos de los recuentos microbiológicos de las muestras de salchichas con L. culinaris, comparadas con los requisitos mínimos establecido en la NTC 1325.

En la formulación utilizada, todos los valores de los análisis microbiológicos estuvieron por debajo de lo establecido en las Normas Técnicas Colombianas (Icontec, 2008).

Los valores de las unidades formadoras de colonias cuantificadas en los análisis de las salchichas fueron, posiblemente de los microorganismos procedentes de la materia prima, condimentos o especias usados que podían contener esporas que no se vieron afectadas por el proceso térmico y que pueden ser causantes del deterioro de los productos finales, si estos no se almacenan adecuadamente. Los coliformes totales se encuentran en el medio ambiente, por tal razón es normal encontrarlos en muchos productos, como los embutidos cárnicos (Granados et al., 2013). En el presente estudio los niveles de estos fueron bajos.

Las materias primas que se utilizaron fueron manejadas bajo normas de calidad para evitar al máximo la contaminación microbiana; los aditivos y demás especias fueron manipulados teniendo la formulación, para así no producir un exceso de estos. Investigaciones realizadas por Hleap, Gutiérrez y Rivera (2010) y Arief et al. (2014), Ilegaron a la conclusión de que para obtener un bajo conteo de microorganismos, se debe recurrir a materias primas frescas y a un buen manejo sanitario, a su vez adecuadas temperaturas en los diferentes tratamientos térmicos, además se debe realizar un rápido enfriamiento del producto y utilizar un empaque apropiado.

En la tabla 5 se muestran los valores obtenidos de los parámetros mecánicos de textura, comparados con la salchicha comercial cuyos componentes se esquematizan en la tabla 3. En los productos cárnicos se considera la dureza, uno de los factores más importantes que determinan la calidad

Tabla 4. Análisis microbiológicos a la salchicha con L. culinaris y comparación con la normatividad

\begin{tabular}{lcc}
\hline \multicolumn{1}{c}{ Parámetros evaluados } & $\begin{array}{c}\text { Valores NTC 1325 } \\
\text { (Log 10 UFC/g) }\end{array}$ & $\begin{array}{c}\text { Salchicha con harina de } \\
\text { L. culinaris (Log 10 UFC/g) }\end{array}$ \\
\hline Aerobios mesófilos & $<4,47 \mathrm{a}$ & $<4,18 \mathrm{~b}$ \\
\hline Coliformes totales & $<2,69 \mathrm{a}$ & $<2,05 \mathrm{~b}$ \\
\hline Coliformes fecales & 0,00 & 0,00 \\
\hline Staphylococcus aureus coagulasa positivo & $<2,00 \mathrm{a}$ & $<1,47 \mathrm{~b}$ \\
\hline Escherichia coli & Ausencia & Negativo \\
\hline Salmonella 25/g & Ausencia & Negativo \\
\hline
\end{tabular}

Fuente: elaboración propia. 
(Torres, González, Acevedo, 2015). En cuanto al análisis del perfil de textura, la salchicha comer-

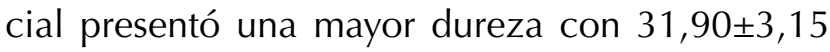
$\mathrm{N}$ en comparación con la salchicha elaborada con harina de L. culinaris $(29,9 \pm 3,80 \mathrm{~N})$; sin embargo esta diferencia no fue estadísticamente significativa $(p>0,05)$. En los embutidos son distintos los resultados de los TPA, ya que depende del tipo de carne utilizada, así como de las materias primas empleadas en la elaboración como grasa, ligantes u extensores (Herrero et al., 2008). Investigaciones como la realizada por Cortes et al. (2010), indican que las salchichas elaboradas únicamente con carne de pollo, fueron menos duras que las fabricadas con carne de pavo, así mismo estos autores concluyen que la incorporación de extensores modifica la dureza de los productos cárnicos.

Por su parte la adhesividad de la salchicha con L. culinaris se encontró dentro del rango reportado por otros autores (Granados, Guzmán y Acevedo, 2013; Herrero et al., 2008), y respecto a la salchicha comercial no hubo diferencias significativas ( $p>0,05)$, lo cual se le atribuye al contenido de humedad similar en estos dos productos.

La cohesividad representa la fuerza con la que están unidas las partículas de un alimento, o el límite hasta el cual se puede deformar un material antes de romperse, es adimensional (Torres, González-Morelo, Acevedo, 2015). La cohesividad de la salchicha realizada con harina de $L$. culinaris fue de 0,28 $\pm 0,07$, lo cual no presentó diferencias estadísticamente significativas respecto a la salchicha comercial $(0,32 \pm 0,08)$, es decir, se requirió menos fuerza para quebrantar los enlaces de la estructura pertenecientes a la matriz de la salchicha elaborada en esta investigación, resultados que coinciden con los reportados por Montero et al. (2015) en la elaboración de un embutido tipo salchicha al cual se le incorporó pasta de ajonjolí y plasma sanguíneo. La elasticidad es el parámetro mecánico de textura que indica qué tanto se ha deformado la estructura de un producto cuando se somete a una fuerza de compresión; así mismo, mide cuánta estructura original del alimento se ha roto, es adimensional, ya que es una longitud dividida por otra (Torres, González-Morelo y Acevedo, 2015). Los resultados obtenidos en esta investigación, señalan que la salchicha con $L$. culinaris fue un producto elástico al igual que el producto control, el cual no presentó diferencias significativas entre los valores hallados $(p>0,05)$.

La masticabilidad representa el trabajo necesario para desintegrar un alimento hasta que esté listo para ser deglutido (Herrero et al., 2008; Montero et al., 2015). En esta investigación se encontró que la masticabilidad para la salchicha comercial fue ligeramente mayor al compararse con la salchicha elaborada con harina de L. culinaris; sin embargo no hubo diferencias significativas entre los dos promedios $(p>0,05)$.

Tabla 5. Análisis de perfil de textura de salchichas con harina de L. culinaris y salchicha comercial

\begin{tabular}{lccc}
\hline \multicolumn{1}{c}{ Parámetros texturales* } & $\begin{array}{c}\text { Salchicha con harina } \\
\text { de } \boldsymbol{L} \text {. culinaris }\end{array}$ & $\begin{array}{c}\text { Salchicha } \\
\text { comercial }\end{array}$ & $\begin{array}{c}\text { Análisis de } \\
\text { Varianza }\end{array}$ \\
\cline { 3 - 4 } & $29,97 \pm 3,80 \mathrm{a}$ & $31,90 \pm 3,15 \mathrm{a}$ & $\mathrm{p}$-valor \\
\hline Dureza $(\mathrm{N})$ & $-0,29 \pm 0,06 \mathrm{a}$ & $-0,23 \pm 0,08 \mathrm{a}$ & 0,09 \\
\hline Adhesividad $(\mathrm{N})$ & $0,28 \pm 0,07 \mathrm{a}$ & $0,32 \pm 0,08 \mathrm{a}$ & 0,27 \\
\hline Cohesividad (adimensional) & $0,92 \pm 0,02 \mathrm{a}$ & $0,95 \pm 0,01 \mathrm{a}$ & 0,43 \\
\hline Elasticidad (adimensional) & $7,72 \pm 1,29 \mathrm{a}$ & $10,28 \pm 2,87 \mathrm{a}$ & 0,35 \\
\hline Masticabilidad $(\mathrm{kg})$ & &
\end{tabular}

*Letras diferentes en una misma fila indican diferencias significativas $(p<0,05)$

Fuente: elaboración propia. 
En general, el comportamiento de los parámetros texturales para la salchicha elaborada con $L$. culinaris en esta investigación fue similar al reportado por Sánchez-Zapata et al. (2011) en un producto cárnico cocido tipo bologna elaborado con pasta de dátil. Los resultados de esta investigación pueden ayudar a entender los cambios en los parámetros de textura de productos cárnicos en los que se adicione este tipo de harina de lenteja de la variedad verdina.

\section{CONCLUSIONES}

El contenido de proteína de la harina fue 35,89 $\%$, mientras que el de fibra fue $11,82 \%$, valores relativamente altos si se comparan con algunas de leguminosas utilizadas para la elaboración de embutidos cárnicos. Los valores de capacidad de retención de agua y absorción de aceite de la harina de lentejas fueron de $3,87 \mathrm{ml}$ agua/g de harina $y$ 2,01 $\mathrm{ml}$ de aceite/g de muestra, estos estuvieron dentro de lo reportados en harinas de leguminosas de uso común. En las salchichas elaboradas con harina de lentejas no se presentaron valores bromatológicos estadísticamente diferentes respecto a las salchichas comerciales, los recuentos microbianos estuvieron dentro de lo reportado por las normas técnicas colombianas. La harina de lenteja influyó positivamente en la aceptabilidad de las salchichas, lo cual fue similar a los obtenidos en las salchichas comerciales, elaboradas con harina de soya. La utilización de harina de L. culinaris fue favorable en todos los parámetros de textura de las salchichas. Es posible el uso de la harina L. culinaris para obtener salchichas de buena calidad y se plantea como una alternativa para la industria alimentaria, en la elaboración de nuevos productos que puedan suplir las necesidades de la población más vulnerable.

\section{FINANCIAMIENTO}

Este estudio fue financiado por los autores en el año 2015, en el desarrollo de actividades de investigación del Grupo Nutrición, Salud y Calidad Alimentaria (NUSCA), adscrito a la Facultad de Ingeniería de la Universidad de Cartagena; a su vez se resalta que el manuscrito fue preparado y revisado con la participación de todos los autores, quienes declaran que no existe ningún conflicto de interés que ponga en riesgo la validez de los resultados.

\section{REFERENCIAS BIBLIOGRÁFICAS}

Achouri, A.; Boye, J.I.; Belanger, D.; Chiron, T.; Yaylayan, V.A. y Yeboah, F.K. (2010). Functional and molecular properties of calcium precipitated soy glycinin and the effect of glycation with k-carrageenan. Food Research International, 43(5), 494-504.

Alvis, A.; Vélez, C.A. y Rada-Mendoza, M. (2008). Composición de ñames frescos cultivados en Colombia y sometidos al freído por inmersión. Información Tecnológica, 19(1), 3-10.

Ambrosiadis, J.; Soultos, N.; Abrahim, A. y Bloukas, J.G. (2004). Physicochemical, microbiological and sensory attributes for the characterization of Greek traditional sausages. Meat Science, 66(2), 279-87.

AOAC International. (2005). Official methods of analysis of AOAC International. Recuperado de: http://www. aoac.org/aoac_prod_imis/AOAC/Publications/ Official_Methods_of_Analysis/AOAC_Member/ Pubs/OMA/AOAC_Official_Methods_of_Analysis.aspx?hkey=5142c478-ab50-4856-8939a7a491756f48

Arief, I.; Wulandari, Z.; Aditia, E.L.; Baihaqi, M. y Noraimah, H. (2014). Physicochemical and Microbiological Properties of Fermented Lamb Sausages Using Probiotic Lactobacillus plantarum IIA-2C12 as Starter Culture. Procedia Environmental Sciences, 20(1), 2-6.

Astaíza, M.; Ruíz, L. y Elizalde, A. (2010). Elaboración de pastas alimenticias enriquecidas a partir de harina de quinua (Chenopodium quinoa wild.) y zanahoria (Daucus carota). Biotecnología en el Sector Agropecuario y Agroindustrial, 8(2), 43-53.

Benjumea, M.V.; Estrada, A. y Álvarez, M.C. (2006). Dualidad de malnutrición en el hogar antioqueño 
(Colombia): bajo peso en los menores de 19 años y exceso de peso en los adultos. Revista Chilena de Nutrición, 33(1), 32-42.

Bermúdez, S. (1994). Preparación y determinación de propiedades funcionales de concentrados proteicos de haba (Vicia faba). Revista Colombiana de Química, 23(1), 4-8.

Boye, J.I.; Aksay, S.; Roufik, S.; Ribéreau, S.; Mondor, M.; Farnworth, E. Y Rajamohamed, S.H. (2010). Comparison of the functional properties of pea, chickpea and lentil protein concentrates processed using ultrafiltration and isoelectric precipitation techniques. Food Research International, 43(2), 37-46.

Castañeda, B.; Manrique, R.; Gamarra, F.; Jáuregui, A.M. y Ramos, F. (2009). Formulación y elaboración preliminar de un yogur mediante sustitución parcial con harina de tarwi (Lupinus mutabilis sweet). Medicina Naturista, 3(1), 5-12.

Cortes, M.; Quezada, E.; Nieto, M.; Alfaro, R.; Güemes, N.; Hernández, J. y Soto, S. (2010). Textura de salchichas elaboradas con mezclas de carne de pavo y pollo. Salud Pública y Nutrición, 20(1), 1-4.

Delgado, N. y Albarracín, W. (2012). Microestructura y propiedades funcionales de harinas de quinua (Chenopodioum Quinoa W) y chachafruto (Erythrina edulis): potenciales extensores cárnicos. Vitae, 9(1), S430-S432.

De la Torre-Gutiérrez, L.; Chel-Guerrero, L.A. y Betancur-Ancona, D. (2008). Functional properties of square banana (Musa balbisiana) starch. Food Chemistry, 106(3), 38-44.

Dzudie, T.; Scher, J. y Hardy, J. (2002). Common bean flour as an extender in beef sausages. Journal of Food Engineering, 52(2), 143-147.

Gharibzahedi, S.M.T.; Emam-Djomeh, Z.; Razavi, S.H. y Jafari, S.M. (2014). Mechanical behavior of lentil seeds in relation to their physicochemical and microstructural characteristics. International Journal of Food Properties, 17(3), 545-558.

Goesaert, H.; Brijs, K.; Veraverbeke, W.S.; Courtin, C.M.; Gebruers, K. y Delcour, J.A (2005). Wheat flour constituents: how they impact bread quality, and how to impact their functionality. Trends in Food Science \& Technology, 6(3), 12-30.
Granito, G.J.; Pérez, D. y Suhey, P. (2009). Valor nutricional y propiedades funcionales de Phaseolus Vulgaris procesada: un ingrediente potencial para alimentos. Interciencia, 34(2), 64-70.

Granados, C., Guzmán, L.E., Acevedo, D. (2013). Análisis proximal, sensorial y de textura de salchichas elaboradas con subproductos de la industria procesadora de atún (scombridae thunnus). Información Tecnológica, 24(6), 29-34.

Guavita, M.J.M. (2012). Gestión del análisis de peligros y puntos críticos de control. Tecnura: Tecnología y Cultura Afirmando el Conocimiento, 16(33), 189-202.

Herrero, A.M.; De la Hoz, L.; Ordóñez, J.A.; Herranz, B.; De Ávila, M.R. y Cambero, M.I. (2008). Tensile properties of cooked meat sausages and their correlation with texture profile analysis (TPA) parameters and physico-chemical characteristics. Meat Science, 80(3), 690-696.

Hleap, J.I.; Gutiérrez, A. y Rivera, L.J. (2010). Análisis microbiológico y sensorial de productos elaborados a partir de surimi de carduma (Cetengraulis mysticetus) y plumuda (Opisthonema spp.). Biotecnología en el Sector Agropecuario y Agroindustrial, $8(4), 57-65$.

Hleap, J.I. y Velasco, V.A. (2012). Parámetros fisicoquímicos durante el almacenamiento de salchichas elaboradas a partir de tilapia roja (Oreochromis sp.). Biotecnología en el Sector Agropecuario y Agroindustrial, 10(1), 42-50.

Jaimes, J.D.C.; Restrepo, D. y Acevedo, D. (2014). Preparación y determinación de las propiedades funcionales del concentrado proteico de trupillo (Prosopis juliflora). Biotecnología en el Sector Agropecuario y Agroindustrial, 12(1), 144-52.

Jaimes, J.D.C.; Torres, J.D. y Severiche, C.A. (2015). Analisis de la calidad de un producto carnico escaldado elaborado con harina de Prosopis juliflora. Ingenium, 9(26), 21-28.

Joshi, M.; Adhikari, B.; Panozzo, J. y Aldred, P. (2010). Water uptake and its impact on the texture of lentils (Lens culinaris). Journal of Food Engineering, 100(1), 61-69. 
Liu, C.; Wang, X.; Ma, H.; Zhang, Z.; Gao, W. y Xiao, L. (2008). Functional properties of protein isolates from soybeans stored under various conditions. Food Chemistry, 111(1), 29-37.

Marchetti, L.; Andrés, S.C. y Califano, A.N. (2014). Low-fat meat sausages with fish oil: Optimization of milk proteins and carrageenan contents using response surface methodology. Meat Science, 96(3), 297-303.

Montero, P.M.; Acevedo, D.; Arnedo, A.J. y Miranda, N.K. (2015). Efecto de la incorporación de plasma sanguíneo y pasta de ajonjolí en la fabricación de un embutido tipo salchicha. Información Tecnológica, 26(6), 55-64.

Instituto Colombiano de Normas Técnicas y Certificación (Icontec). NTC 1325. Norma colombiana sobre productos cárnicos no enlatados. Bogotá.

Olmedilla-Alonso, B.; Farré-Rovir, R.; Asensio-Vegas, C. y Martín-Pedrosa, M. (2010). Papel de las leguminosas en la alimentación actual. Actividad Dietética, 14(2), 72-6.

Olmedilla-Alonso, B.; Jiménez-Colmenero, F. y Sánchez-Muniz, F.J. (2013). Development and assessment of healthy properties of meat and meat products designed as functional foods. Meat Science, 95(4), 919-930.

Oroian, M. (2015). The temperature hydration kinetics of Lens culinaris. Journal of the Saudi Society of Agricultural Sciences. In press, 1-7.

Ortega-David, E.; Rodríguez, A. y Zamora-Burbano, Á. (2010). Caracterización de semillas de lupino (Lupinus mutabilis) sembrado en los Andes de Colombia. Acta Agronómica, 59(1), 111-118.

Oshodi, A.A. y Ekperigin, M.M. (1989). Functional properties of pigeon pea (Cajanus cajan) flour. Food Chemistry, 34(3), 187-91.
Restrepo, S.L.; Mancilla, L.P.; Parra, B.E.; Manjarrés, L.M.; Zapata, N.J.; Restrepo-Ochoa, P.A. y Martínez, M. I. (2010). Evaluación del estado nutricional de mujeres gestantes que participaron de un programa de alimentación y nutrición. Revista Chilena de Nutrición, 37(2), 18-30.

Rodríguez, V.; Amorrortu, I. y Álvarez, M.J. (2011). Setting parameters in the cold chain. Tecnura, 15(30), 71-81.

Sánchez-Zapata, E.; Fernández-López, J.; Peñaranda, M.; Fuentes-Zaragoza, E.; Sendra, E.; Sayas, E. y Pérez-Alvarez, J.A. (2011). Technological properties of date paste obtained from date by-products and its effect on the quality of a cooked meat product. Food Research International, 44(7), 2401-2407.

Torres, J.D.; Acevedo, D. y Tirado, D.F. (2015). Análisis de la calidad bromatológica, microbiológica, sensorial y de textura de bollos de mazorca cocidos en ebullición. Revista RECITEIA, 14(2), 7-16.

Torres, J.D.; González-Morelo K. y Acevedo D. (2015). Análisis del perfil de textura en frutas, productos cárnicos y quesos. Revista RECITEIA: Revisiones de la Ciencia, Tecnología e Ingeniería de los Alimentos, 14(2), 63-75.

Umaña, J.; Lopera, S. y Gallardo, C. (2013). Caracterización de harinas alternativas de origen vegetal con potencial aplicación en la formulación. Alimentos Hoy, 22(29), 33-46.

Utrilla, M.C.; García-Ruiz, A. y Soriano, A. (2014). Effect of partial reduction of pork meat on the physicochemical and sensory quality of dry ripened sausages: Development of a healthy venison salchichon. Meat Science, 98(4), 85-91.

Zhang, W.; Xiao, S.; Samaraweera, H.; Lee, E.J. y Ahn, D.U. (2010). Improving functional value of meat products. Meat Science, 86(1), 15-31.

\section{(C) $(1) \Theta$}

